\title{
Sources of geomagnetic activity during nearly three solar cycles (1972-2000)
}

\author{
I. G. Richardson' ${ }^{1}$ and H. V. Cane ${ }^{2}$ \\ NASA Goddard Space Flight Center, Greenbelt, Maryland, USA
}

E. W. Cliver

Air Force Research Laboratory, Hanscom Air Force Basc, Bedford, Massachusetts, USA

Received 27 September 2001; revised 7 December 2001; accepted 9 January 2002; published XX Month 2002.

[1] We examine the contributions of the principal solar wind components (corotating highspeed streams, slow solar wind, and transient structures, i.e., interplanetary coronal mass ejections (CMEs), shocks, and postshock flows) to averages of the a a geomagnetic
index and the interplanetary magnetic field (IMF) strength in 1972-2000 during nearly three solar cycles. A prime motivation is to understand the influence of solar cycle variations in solar wind structure on long-term (e.g., approximately annual) averages of these parameters. We show that high-speed streams account for approximately two-thirds of long-term $a a$ averages at solar minimum, while at solar maximum, structures associated with transients make the largest contribution $(\sim 50 \%)$, though contributions from streams and slow solar wind continue to be present. Similarly, high-speed streams are the principal contributor $(\sim 55 \%)$ to solar minimum averages of the IMF, while transientrelated structures are the leading contributor $(\sim 40 \%)$ at solar maximum. These differences between solar maximum and minimum reflect the changing structure of the near-ecliptic solar wind during the solar cycle. For minimum periods, the Earth is embedded in high-speed streams $\sim 55 \%$ of the time versus $\sim 35 \%$ for slow solar wind and $\sim 10 \%$ for CME-associated structures, while at solar maximum, typical percentages are as follows: high-speed streams $\sim 35 \%$, slow solar wind $\sim 30 \%$, and CME-associated $\sim 35 \%$. These compositions show little cycle-to-cycle variation, at least for the interval considered in this paper. Despite the change in the occurrences of different types of solar wind over the solar cycle (and less significant changes from cycle to cycle), overall, variations in the averages of the $a a$ index and IMF closely follow those in corotating streams. Considering solar cycle averages, we show that high-speed streams account for $\sim 44 \%, \sim 48 \%$, and $\sim 40 \%$ of the solar wind composition, $a a$, and the IMF strength, respectively, with corresponding figures of $\sim 22 \%, \sim 32 \%$, and $\sim 25 \%$ for CME-related structures, and $\sim 33 \%$, $\sim 19 \%$, and $\sim 33 \%$ for slow solar wind. INDEXTERMS: 2134 Interplanetary Physics: Interplanetary magnetic fields; 2162 Interplanetary Physics: Solar cycle variations (7536); 2164 Interplanetary Physics: Solar wind plasma; 2111 Interplanetary Physics: Ejecta, driver gases, and magnetic clouds; 2788 Magnetospheric Physics: Storms and substorms; KEYWORDS: geomagnetic activity, solar cycle variation, solar wind, interplanetary magnetic field

\section{Introduction}

[2] Studies of long-term trends in space weather typically use extended (often yearly) averages of data such as geomagnetic indices and solar wind parameters [e.g., Feynman and Crooker, 1978; Bounar et al., 1997; Cliver et al., 1998; Ahluwalia, 1999; Hathaway et al., 1999; Kishcha et al., 1999; Lockwood et al., 1999; Stamper et al.,

\footnotetext{
'Also at Department of Astronomy, University of Maryland, College Park, Maryland, USA.

${ }^{2}$ Also at School of Mathematics and Physics, University of Tasmania, Hobart, Australia.

Copyright 2002 by the American Geophysical Union. 0148-0227/02/2001 JA000504\$09.00
}

1999; Lockwood and Foster, 2000; Lockwood, 2001]. Such extended averages are inherently complex since they include contributions from different types of solar wind structures. These structures include transients (shocks and interplanetary coronal mass ejections), which may generate large geomagnetic storms [e.g., Gosling et al., 1991; Richardson et al., 2001], corotating high-speed streams, which produce intervals of moderately-enhanced geomagnetic activity extending over several days recurring with the solar rotation period [e.g., Crooker and Cliver, 1994; Tsurutani et al., 1995], and slow solar wind, which typically is associated with low activity levels [e.g., Richardson et al., 2001]. Within each solar wind structure, the parameters may vary significantly on time-scales of hours or less.

SHP $\quad X-1$ 
[3] Our previous study of the period 1972-1986, including solar cycle 21 [Richardson et al., 2000], quantificd the relative contributions to three-solar-rotation averages of the aa geomagnetic index [Mayaud, 1972], the solar wind speed, and the IMF from different types of solar wind structures and showed that these contributions do vary throughout the solar cycle. The $a a$ index was chosen because it extends further back in time (to 1868) than other geomagnetic indices and is frequently used in long-term studies of geomagnetic activity. Corotating streams provide the dominant contributions to $a a$ and the solar wind parameters at solar minimum. Though there is an increasing contribution from transients at higher levels of solar activity, the widespread assumption that geomagnetic activity at the peak of the cycle is predominantly caused by CMEs is incorrect. While it is certainly true that most intense activity is associated with CMEs, even at solar maximum, significant contributions are made to averaged activity indices by corotating streams and slow solar wind. Overall, we found that long-term averages of both $a a$ and the IMF strength tended to follow average values in the background solar wind (in particular corotating high-speed streams) through cycle 21 . In the present paper, we will consider a period extending up to the near present (1972-2000), including nearly three solar cycles, in order to verify and extend the conclusions of our previous study. We will also summarize the cycle-to-cycle variations in the contributions of the various types of solar wind around solar minimum and maximum and averaged over each cycle.

\section{Solar Wind Structure Classification}

[4] As described in detail by Richardson et al. [2000], we used 1-hour averaged near-Earth solar wind plasma and magnetic field data from the National Space Science Data Center (OMNI) database to classify the solar wind during $1972-2000$ into four categories: "CME-associated" (i.e., including interplanetary coronal mass ejections (ICMEs), the transient forward shocks generated ahead of fast ICMEs, and the related postshock flows); corotating high-speed solar wind streams from coronal holes and associated corotating interaction regions; slow, interstream solar wind; and "uncertain" (e.g., insufficient data were available to classify the type of solar wind present, or we were unable to include it in another category). Our initial study [Richardson et al., 2000] covered the period 1972-1986 when the OMNI data coverage is reasonably complete. After this time until the launch of the WIND spacecraft in November 1994, there are intermittent gaps, typically of several days duration, because the OMNI data were provided by IMP 8 , which was located in the solar wind for only $\sim 60 \%$ of each $\sim 12.5$ day orbit. For more recent periods, the OMNI data base incorporates data from the WIND and Advanced Composition Explorer (ACE) spacecraft, which provide nearly continuous solar wind observations. We finish our analysis at the end of 2000 because at the time of writing, there are only limited OMNI data available for 2001.

[s] To complement the in-situ solar wind data, and to help infer the solar wind structures present when no solar wind data were available, we have referred to other data sets. For example, geomagnetic storm sudden commencements help to identify interplanetary shocks where the in situ observations are incomplete. Solar energetic particle events can help identify shocks and ejecta associated with energetic solar events/CMEs, while cosmic ray intensity modulations (i.e., Forbush decreases) observed by spacecraft (e.g. IMPs 7/8) and neutron monitors can indicate the passage of these structures past the Earth [e.g., Cane et al., 1994]. Energetic particle intensity variations can also be used to help identify corotating streams [e.g., Richardson et al., 1998, and references therein].

[6] Our classification of the solar wind into the categories described above is based upon recognizing the signatures of various types of solar wind flow. These signatures have been described in a number of previous works. The solar wind signatures of corotating streams/corotating interaction regions are described by Belcher and Davis [1971]. To identify ICMEs, we examined available observations of a range of typical ICME signatures [e.g., Gosling, 1990; Richardson et al., 2000, and references therein] including magnetic clouds, intervals of bidirectional solar wind electron heat fluxes, bidirectional energetic $\sim 1 \mathrm{MeV}$ ions, solar wind helium abundance enhancements, intervals of abnormally low plasma proton temperature $T_{p}$ and the cosmic ray and energetic particle depressions which are typically associated with ICMEs. In general, we find that most ICMEs, in particular those important from the point of view of geomagnetic activity, are well indicated by several signatures. It would have been interesting to sub-divide "CME-associated" regions into ICMEs and other postshock flows. However, we chose not to do so because we cannot distinguish these structures unambiguously during sections of our study interval due to the incomplete and uneven solar wind data coverage. (For the same reason, we do not attempt here to separate out the corotating stream contribution associated with corotating interaction regions.) Slow solar wind regions have solar wind speeds below $\sim 400 \mathrm{~km} / \mathrm{s}$, typically are relatively dense, and include wide variations in the plasma parameters.

\section{Analysis}

\subsection{Relationship Between aa and Solar Wind} Structures

[7] We will first discuss the relationship between $a a$ and solar wind structures. Figure 1 summarizes the average aa values associated with the various solar wind regions in 1972-2000. This period encompasses the decline of solar cycle 20 through to the maximum of cycle 23 . The monthly mean sunspot number for this period is given in Figure la. Figure $\mathrm{lb}$ shows the average $a a$ index $(\overline{a a})$. We calculate $3-$ solar (Carrington) rotation averages of $a a$ because these show both long-term trends and features at a finer scale than the yearly-averages typically used in long-term studies. Overall, $\overline{a a}$ is rather poorly correlated with the sunspot number through the solar cycle (correlation coefficient $(c c)$ $=0.306$ for the period in Figure 1). One reason is that although there is a tendency for $\bar{a} a$ to increase as solar activity rises, there is often a temporary decrease in geomagnetic activity near sunspot maximum, as indicated by the arrows in Figure $1 \mathrm{~b}$. This decrease is particularly conspicuous in 1980 at the maximum of cycle 21 , when $\bar{a} \bar{a}$ declined to some of the lowest values observed during the period in Figure 1. Another activity decrease is evident 
RICHARDSON ET AL:: SOURCES OF GEOMAGNETIC ACTIVITY $(1972-2000)$ SHP $\quad$ X -3

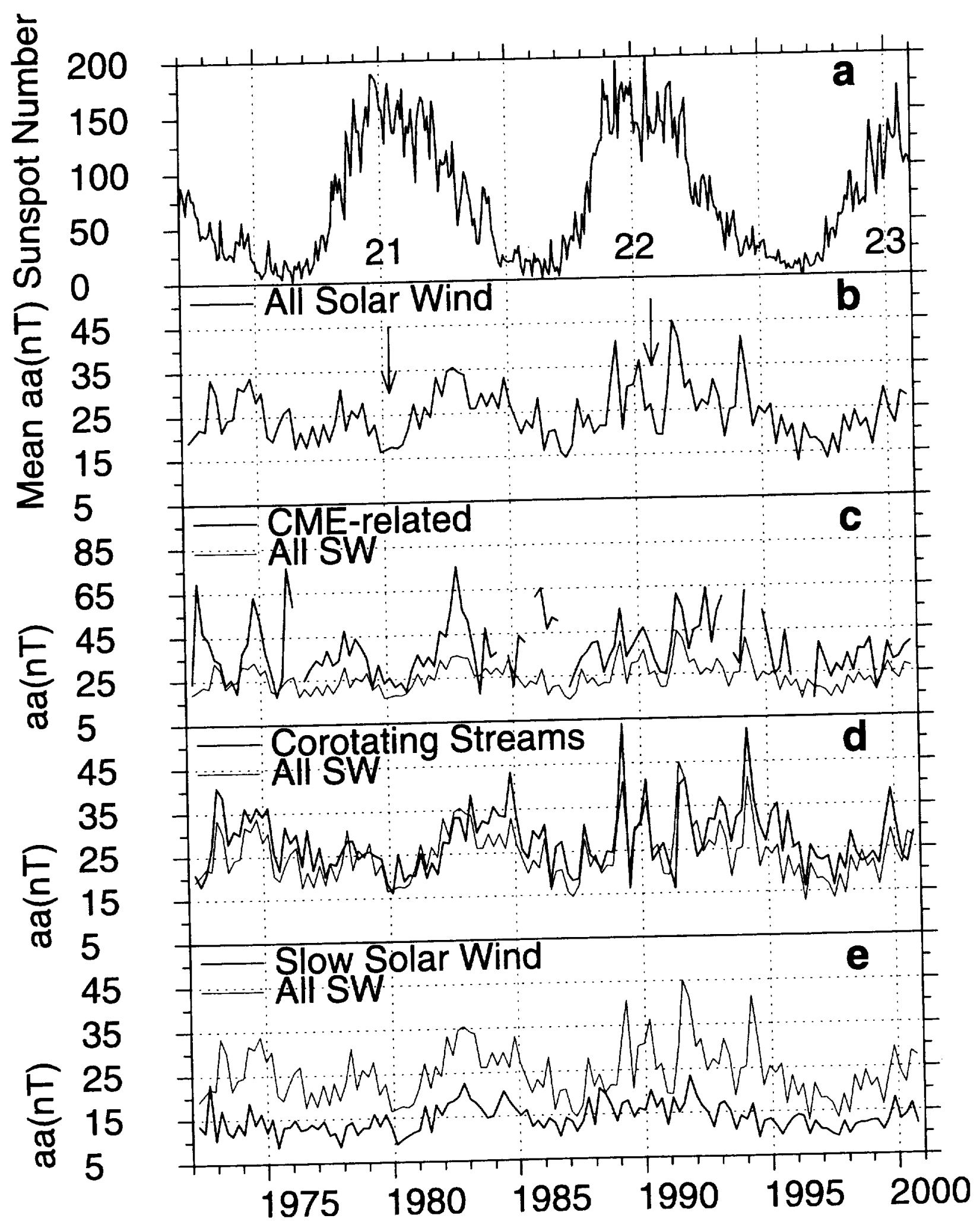


in 1990-1991 near the maximum of cycle 22. As discussed by Richardson et al. [2000], such decreases are most likely manifestations of what has been termed the "Gnevyshev Gap" by Feminella and Storini [1997] [see also Gnevyshev, $1967,1977]$, resulting from a temporary reduction in the rate of energetic phenomena at the Sun at the time of the reversal of the solar magnetic field near sunspot maximum. The data for cycle 23 shown in Figure 1 suggest that this temporary reduction in aa was not present before the end of 2000. (However, preliminary aa data indicate that this feature occurred in January-September 2001, when rotation-averages of $a a$ were predominantly in the range $\sim 15-$ $20 \mathrm{nT}$ compared to $\sim 25 \mathrm{nT}$ in the preceding year.) We note that, because these temporary depressions last of the order of 1 year, their phasing may significantly influence yearly averages of $a a$ near solar maximum. Another difference between the time profiles of $\overline{a a}$ and the sunspot number is the tendency for $\bar{a}$ to be enhanced during the declining phase of each solar cycle. This is especially evident during the decline of cycle 20 when there was a $\sim 2$-year period (1973-74) during which $\overline{a a}$ was between $\sim 25-34 \mathrm{nT}$ and the sunspot number was < 50 .

[8] Figures $1 \mathrm{c}-\mathrm{le}$ show the mean values of $a a$ in each solar wind region ("CME-related", i.e. shocks, postshock flows and ICMEs; corotating streams; and slow solar wind). The value of $\bar{a} a$ for all solar wind regions is repeated in each figure for comparison. As might be expected, since they generate most major geomagnetic storms, CME-related structures are associated with higher than average activity levels ( $a a \sim 25-75 \mathrm{nT}$; note the extended vertical scale in Figure $1 \mathrm{c}$ ). The large variability in $a a$ averages for CMErelated flows is particularly evident at lower solar activity levels when there are fewer CME-related structures, because a given CME-related structure does not necessarily generate enhanced geomagnetic activity [Gosling et al., 1991; Richardson et al., 2001]. (For example, it may not include enhanced southward-directed magnetic fields [Cane et al., 2000 ].) Activity levels in corotating streams ( $a a \sim 15-45 \mathrm{nT})$ track $\bar{a} a$ for all solar wind remarkably closely throughout the period in Figure $1(c c=0.79)$. Slow solar wind generates lower than average activity levels ( $a a \sim 9-24 \mathrm{nT}$ ), though temporal variations again tend to follow those seen in $\overline{a a}(c c=$ $0.65)$. Even activity levels in CME-related structures, though more variable, also show a significant correlation with $\overline{a a}(c c=0.60)$. Note that the Gnevyshev gap in $a a$ appears to be present in all solar wind regions.

[9] Figure 2 shows, for the same period as Figure 1, the fraction (in \%) of $\bar{a} a$ contributed by each type of stream structure. (Note that the contribution of "unclear" intervals (Figure $2 \mathrm{~d}$ ) increases in cycle 22 because of the intermittent solar wind data coverage.) The relative contributions to $\overline{a a}$ from $\mathrm{CME}$-associated structures and corotating high- speed streams clearly vary with the phase of the solar cycle. Corotating streams provide the major contribution around solar minimum, with little contribution at this time from CME-related structures. Around solar maximum, CME-related structures are the largest contributor to $a a$ though there continue to be important contributions from the other types of solar wind. A major influence in these varying contributions is the amount of time spent in each type of structure, shown in Figure 3, with CME-related structures (Figure 3a) occupying only $<\sim 10 \%$ of the solar wind at solar minimum but $\sim 35 \%$ of the solar wind around solar maximum. There is a corresponding change in the corotating high-speed stream component (Figure 3b) from $\sim 55 \%$ of the solar wind around solar minimum to $\sim 35 \%$ of the solar wind at solar maximum. Slow solar wind (Figure 3c) contributes $\sim 30 \%$ of the solar wind throughout the solar cycle. In section 4 , we will summarize further the changes in solar wind structure during the solar cycle, as well as cycle-to-cycle variations in the contributions of the various flow types to averages of aa and solar wind parameters.

\subsection{Relationship Between Solar Wind Structures, the Solar Wind Speed, and IMF}

[10] The contribution of the three basic types of solar wind flows to average aa values is determined by the relative "geoeffectiveness" of these flows, as well as by the time the Earth spends in each flow type. Previous studies [e.g., Feynman and Crooker, 1978] suggest that $a a$ is highly correlated with $V^{2} B_{s}$ in the solar wind encountering the Earth. It is therefore of interest to examine how the solar wind speed, IMF, and $B_{s}$ vary in each type of solar wind structure over the 1972-2000 interval and how they influence the geoeffectiveness.

[11] Figure 4 shows variations in the solar wind speed in (a) all solar wind, (b) CME-related structures (c) and corotating high-speed streams in 1972-2000. Average solar wind speeds tend to be highest prior to solar minimum, when corotating streams are dominant (see Figure 3). Around solar maximum, as noted by Richardson et al. [2000] for cycle 21, solar wind speeds associated with both streams and CMEs tend to show minima at the time of the Gnevyshev gap near solar maximum (e.g., 1980 and $\sim 1990$ ). However, there are also clear variations from cycle to cycle. High speeds associated with streams were prominent and extended during the declining phase of cycle 20 $(\sim 1973-1976)$ and were associated with a sequence of "monster" coronal holes [Hundhausen, 1977; Feynman, 1980]. During the decline of cycle $21(\sim 1984-87)$ highspeed streams were also present. Though they appear to have been less persistent than in cycle 20 , this conclusion may be affected by incomplete data coverage. The declining

Figure 1. (opposite) Three-solar rotation averages of the aa geomagnetic index during 1972-2000 (b-e), together with the monthly sunspot number (a). The period shown extends from the trailing edge of solar cycle 20 to near the maximum of cycle 23. The mean $a a$ index for all solar wind regions is shown in (b). Figures c-e show respectively the mean $a a$ in $\mathrm{CME}$-related structures, corotating streams and low speed solar wind. The mean aa for all solar wind regions is repeated in each of these figures for comparison. This follows activity levels in corotating streams most closely. CMErelated structures are associated with higher than average geomagnetic activity, while slow solar wind has lower than average activity. The arrows in (b) indicate temporary decreases in aa right at solar maximum which are a manifestation of the "Gnevyshev Gap". 


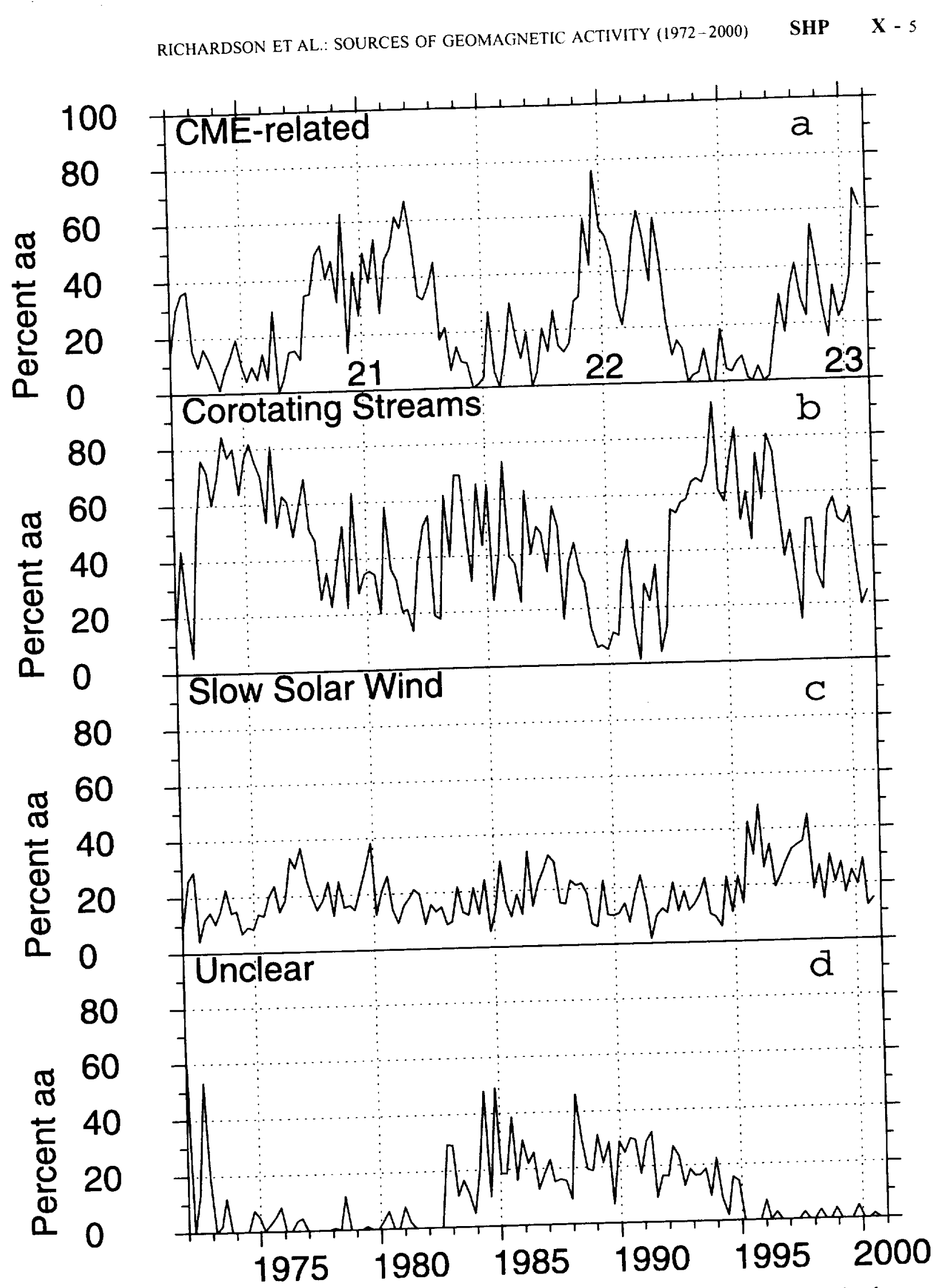

Figure 2. Fraction (\%) of 3-rotation averages of $a a$ in 1972-2000 contributed by (a) CME-related structures, (b) corotating streams, (c) slow solar wind, and (d) intervals where the type of solar wind structure is unclear. 


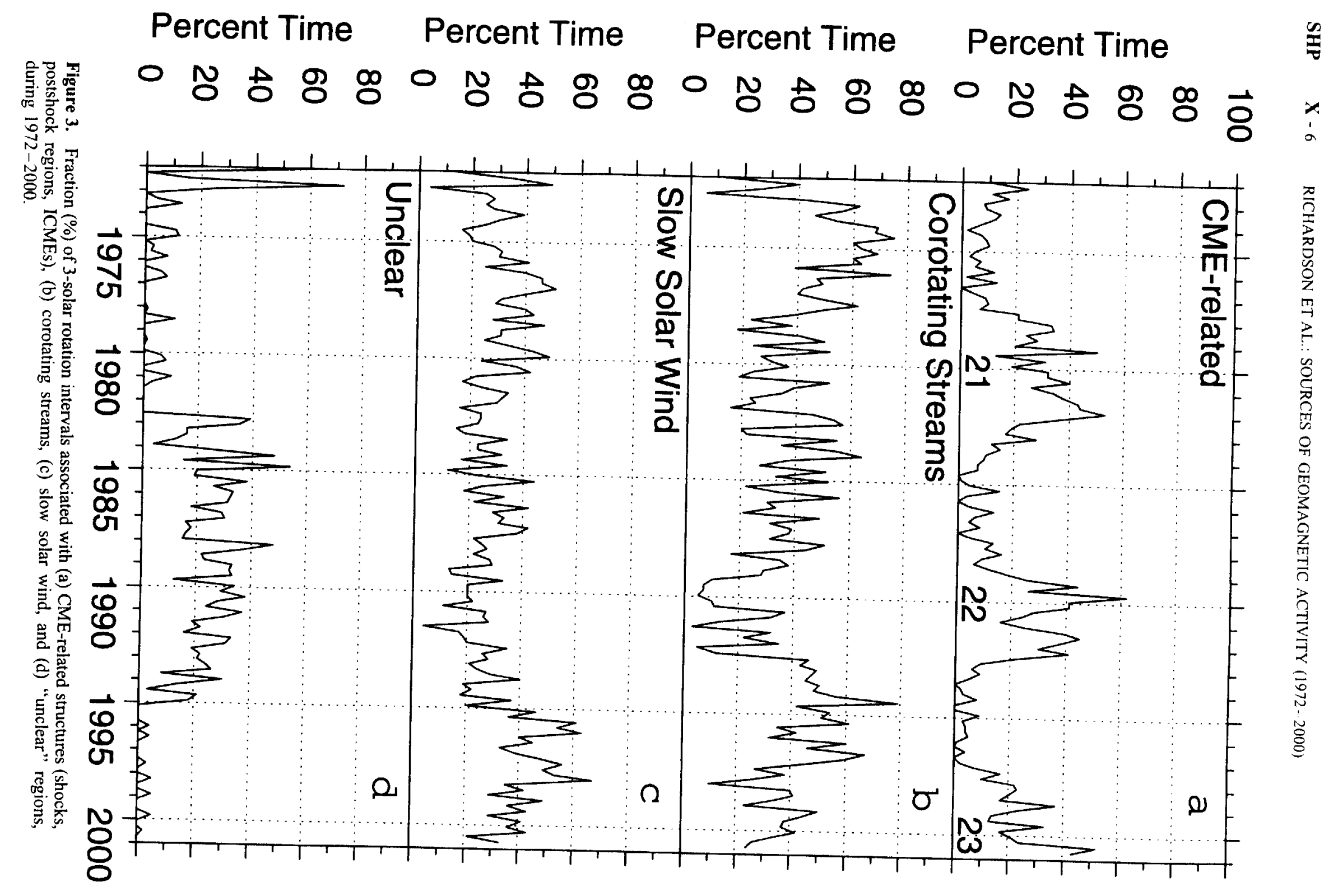




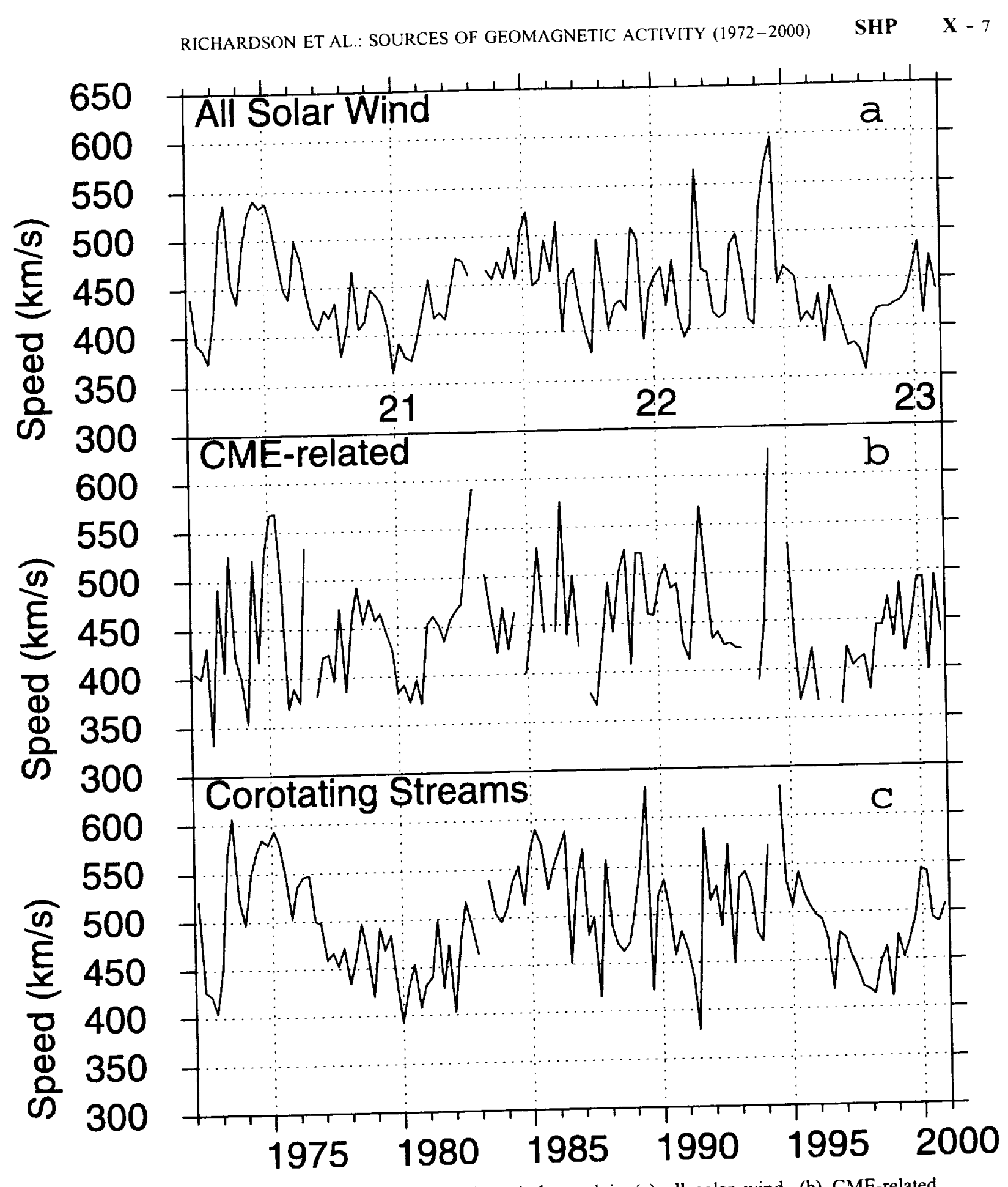

Figure 4. Three-rotation averages of the solar wind speed in (a) all solar wind, (b) CME-related structures, and (c) corotating streams, in 1972-2000.

phase of cycle 22 shows a rather brief burst of high-speed flows in 1994 (corotating flows and fast CMEs) compared to that observed two cycles earlier. This was followed in 1995 by a transition to a period of exceptionally slow corotating flows near solar minimum (some of which might be classified as slow solar wind except for clear signatures of corotating interaction regions), then an increase in solar wind speed from 1998 during the ascending phase of cycle
23. This pattern was not seen in the previous two cycles, which showed more of a general decline in solar wind speed from $\sim 550 \mathrm{~km} / \mathrm{s}$ to $\sim 450 \mathrm{~km} / \mathrm{s}$ from the declining phase of one cycle to the maximum of the next (e.g., 1975-1980 and 1985 -1990). The cycle-to-cycle variations in the solar wind stream structure presumably arise from differences in the configuration of the source coronal holes in each cycle [see Luhmann et al., 2002]. 
[12] Overall, as for $a a$, inspection of Figure 4 suggests that solar cycle variations in the mean solar wind speed follow most closely variations in the speed of corotating streams, largely because of the predominance of streams prior to solar minimum. At solar maximum, even though CME-related structures are a more prominent component of the solar wind, their average speeds at $1 \mathrm{AU}$ are comparable or even lower than those of streams, consistent with previous observations suggesting that the speeds of ICMEs at $1 \mathrm{AU}$ tend to converge toward that of the background solar wind [e.g., Gosling et al., 1987; Lindsay et al., 1999]. The tendency for the speeds of the (infrequent) CME-related structures near solar minimum, when high-speed streams are predominant, to be higher than those observed at solar maximum may also be associated with this convergence.

[13] There are clearly features in $a a$ (Figure 1) associated with variations in the solar wind speed such as the high aa values during the declining phase of the solar cycle (notably in 1973-1975 and 1994). The difference in speed profiles during the preceding minima and ascending phases of cycles 21 and 23 is also reflected in $a a$, with the lowest aa values for cycle 23 occurring during minimum conditions (apparently associated with the extended period of low solar wind speeds), whereas in cycle 21 , the lowest values of $a a$ and the solar wind speed occurred near solar maximum. However, the correlations between $a a$ and the solar wind speed are rather poor (for example the correlation coefficient for all solar wind is 0.36 ).

[14] We now consider the variations in long-term averages of the IMF in each type of solar wind structure and their relationship with aa. Although we show here $|B|$ rather than $B_{s}$, which is the most important component for generating geomagnetic activity, temporal variations in $B_{s}$ are similar to those in $|B|$ (e.g., $c c$ (all solar wind) $=0.76$ ). During the study period, $B_{s} \sim 0.16|B|$ in streams and slow solar wind. The $B-B_{s}$ correlation is poorer for CME-related structures $(c c=0.45)$ and the best fit indicates $B_{s} \sim 0.23|B|$, consistent with transient structures having relatively larger out-of-the-ecliptic freld components [e.g., Slavin and Smith, 1983].

[15] Figures $5 c-5 f$ show the average magnetic field strengths observed in all solar wind regions and in CMErelated structures, corotating streams, and slow solar wind separately. The mean field for all solar wind regions (Figure $5 \mathrm{c}$, also repeated in Figures $5 \mathrm{~d}-5 \mathrm{f}$ for comparison) shows the well-known solar cycle variation in the strength of the IMF, tending to be enhanced as solar activity levels increase [e.g., Slavin and Smith, 1983; Cane et al., 1999]. The Gnevyshev Gap effect in the IMF is evident near the maxima of solar cycles 21 and 22 (indicated by the arrows in Figure $5 \mathrm{c}$ ). As was the case for $a a$, we see that the mean field for all solar wind tracks the mean fields in corotating streams (Figure 5e) very closely. Slow solar wind also shows similar variations in mean field (Figure 5f). CME-associated fields (Figure 5d) are stronger than average, but the enhancement is more evident at times of lower solar activity levels when there are few CMEs and the background IMF is weaker. Around solar maximum, average fields in CME-related structures are only modestly $(\sim 30 \%)$ above those elsewhere in the solar wind.

[16] An important conclusion from Figure 5 is that the solar cycle variation in the average IMF strength reflects changes in magnetic field strength in the "quasi-stationary" solar wind structures (slow solar wind and corotating highspeed streams) and is not solely driven by the injection of high fields associated with CMEs. Wang et al. [2000] show that variations in $\left|B_{r}\right|$ are closely correlated with the open flux at the Sun calculated from the observed photospheric field and a potential field model. Their calculated open flux is reproduced in Figure 5a. The variations in the total IMF strength and the open flux are also similar, both showing a tendency to increase with solar activity levels, the Gnevyshev Gap effect, and enhancements of $\sim 1$ year duration that occur throughout the period in Figure 5. The calculated open flux tends to decline more slowly during the declining phase of the solar cycle (in particular in 1985-88) though this may be an instrumental effect caused by the difficulty of measuring fields at high latitudes [Wang et al, $2000]$. The higher background fields at solar maximum are associated with low latitude, active region associated, coronal holes which have stronger intrinsic magnetic fields than the high-latitude coronal holes that contribute near solar minimum [e.g., Wang et al., 1996, 2000; Luhmann et al., 2002].

[17] Figure 5b shows $\bar{a} \bar{a}$ for all solar wind regions (reproduced from Figure $\mathrm{lb}$ ). Despite the complexity of $\overline{a a}$ noted above, inspection indicates that there is clearly a high degree of correlation $(c c=0.66)$ between the average IMF strength and $\overline{a a}$. In particular, both show the tendency to increase with solar activity levels, with a temporary decrease near solar maximum. There are also individual features in the profiles, in particular around solar maximum, which may be associated and related to variations in the open flux. There are also some differences, such as the $\overline{a a}$ enhancement in $1973-4$ associated with high-speed streams as discussed above. $\overline{a a}$ also tends to be correlated with variations in the open flux [see also Wang et al., 2000].

[18] We now examine the relationship between $a a$ and $V^{2} B_{s}$, in particular whether this relationship is a universal one, being similar in each solar wind region. Figure 6 shows a scatterplot of 3-rotation averages of $a a$ and $V^{2} B_{s}$ in each type of solar wind structure. This is consistent with variations in $V^{2} B_{s}$ being the major factor determining the geoeffectiveness of each type of solar wind. The best fit lines are similar for streams and slow solar wind, indicating that $a a$ and $V^{2} B_{s}$ are similarly related in both types of solar wind structure. The data points for CMErelated structures are more scattered, though the best fit is again similar. The larger scatter for CME-related structures indicates that three-rotation averages of the solar wind parameters are not particularly accurate indicators of the level of transient geomagnetic activity. Another effect which introduces scatter in Figure 6 , is that averages of $a a$ or $V^{2} B_{s}$ during a given 3-rotation period will not be derived from exactly the same intervals of data if there are gaps in the solar wind data ( $a a$ has no gaps). An extreme example in Figure 6 is the CME-related average with $a a=61 \mathrm{nT}$ and $V^{2} B_{s}=0$. This arises because the interval of simultaneous field and solar wind speed observations during this 3-rotation period was limited and did not include significant southward magnetic fields, whereas $a a$ is higher than average, presumably because solar wind with enhanced values of $V^{2} B_{s}$ encountered the 


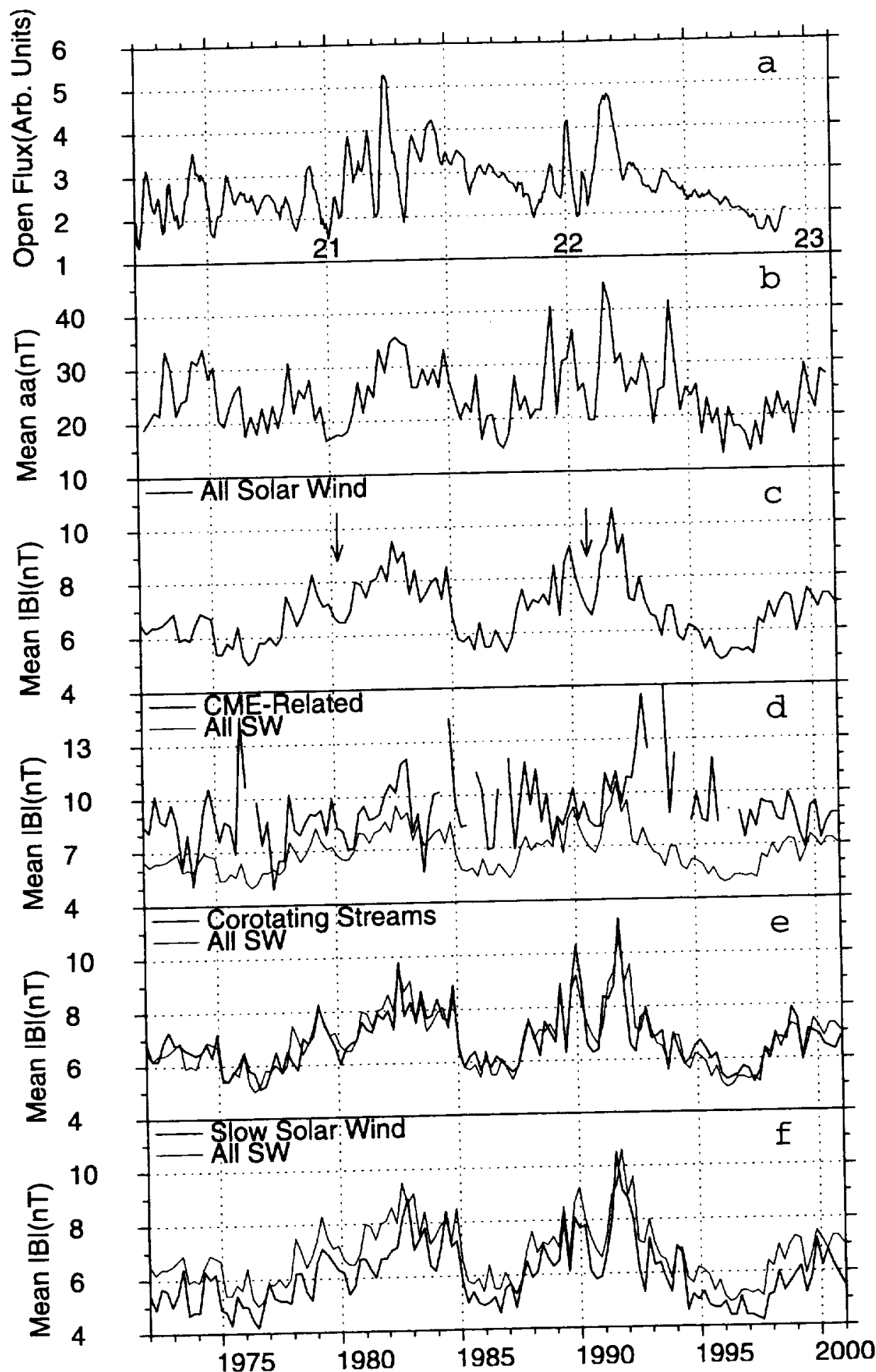

Figure 5. (a) shows the open flux at the Sun (courtesy of Y.-M. Wang) and (b) the mean aa index (from Figure 1 b). (c-f) show the variation of the IMF strength (3-solar rotation averages) during 1972-2000 in all solar wind regions (c), CME-related regions (d), corotating streams (e) and slow solar wind (f), the average in all solar wind regions being repeated in Figures $d-f$. The Gnevyshev Gap at solar maximum is indicated by arrows in (c). 


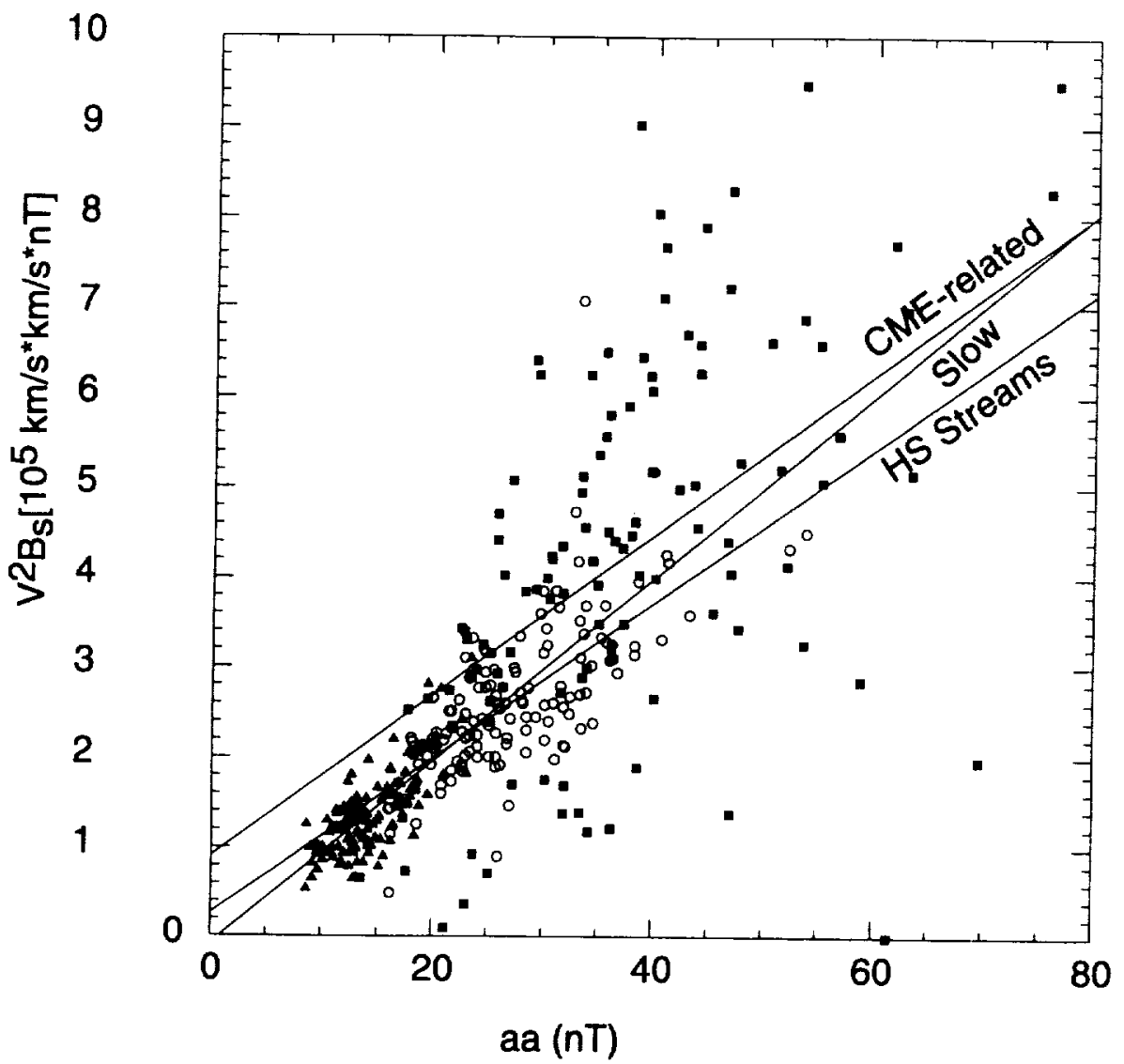

Figure 6. Scatterplot of 3-rotation averages of $V^{2} B_{s}$ in CME-related structures (solid squares), corotating streams (open circles), and slow solar wind (solid triangles) in 1972-2000, plotted against the average $a a$ in the same structures.

Earth at times when no solar wind observations were being made.

\section{Summary of Solar Cycle Variations}

[19] In addition to the 3-rotation averages considered above, it is useful to summarize the differences between solar maximum and minimum and from cycle to cycle in solar wind structure and the contributions of the various solar wind types to geomagnetic activity ( $a a)$, and IMF averages. Figure 7a illustrates, for three intervals around solar minimum (sunspot number generally $<\sim 50$ ) during our study period (1973-1977, 1983-1987, and 1993-1997), the fraction of the time the solar wind was occupied by each type of structure, together with the relative contributions to average $a a$ and the magnetic field strength made by each type of structure. Figure $7 \mathrm{~b}$ shows the same information for three "solar maximum" intervals (1978-1982, 1988-1992 and 1998-2000; note that the last interval includes only a partial maximum). In these plots, the "unclear" intervals have been removed, i.e., it is assumed that they contained a similar mix of structure types as the remainder of the solar wind. The results are summarized in Table 1 which also gives the average $a a$, IMF strength and solar wind speed in the various types of solar wind for each interval, as well as similar parameters averaged over all the solar minimum or solar maximum intervals, and cycles 21 or 22 .

[20] The change in the structure of the solar wind from solar minimum to solar maximum noted in section 3 is very apparent in Figure 7 (see also Table 1). The fraction of time associated with CME-related structures increases from $\sim$ one tenth at solar minimum to $\sim$ one third at solar maximum, while there is a reduction in the high-speed stream fraction from $\sim 55 \%$ to $\sim$ one third. On average, the slow solar wind component remains at around one third at both solar maximum and minimum, although for the recent 1993-1997 minimum the fraction of time Earth spent in the slow solar wind exceeded $40 \%$. Note that the solar wind composition is relatively constant from cycle 21 to cycle 22 (Table 1), most likely because these cycles have similar sizes. (The slightly reduced CME-related fraction in the current solar maximum is consistent with the maximum sunspot number being lower than in the two preceding cycles (Figure 1a). The corresponding changes in the contributions to $\overline{a a}$ and $|B|$ are also evident. For example, CMEs-related structures contribute $\sim 50 \%$ of $\overline{a a}$ at solar maximum, and $\sim 10 \%$ at solar minimum. Corotating streams contribute $\sim$ two-thirds of $\overline{a a}$ at solar minimum, and continue to make a contribution of $\sim 30 \%$ at solar maximum. Slow solar wind makes a contribution of $\sim 20 \%$ 
a

Solar Minimum: Percent. Time

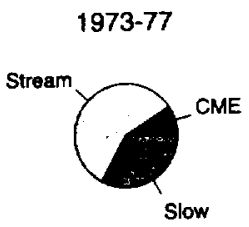

1983-87
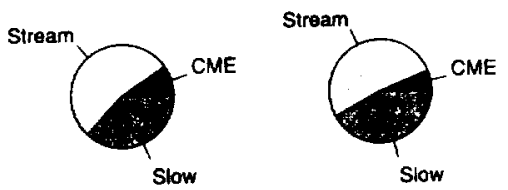

Percent. aa

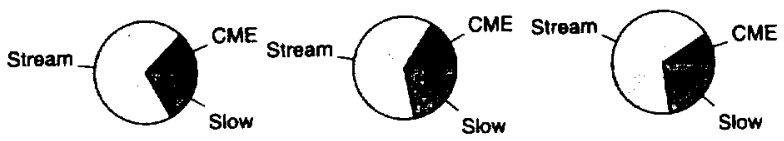

Percent. |B|
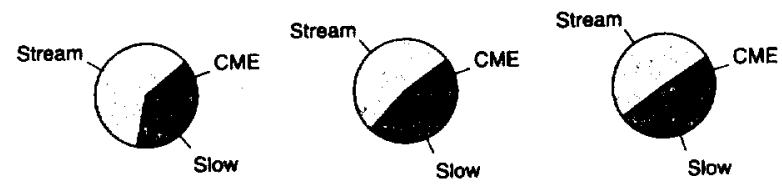

b Solar Maximum: Percent. TIme 1978-82

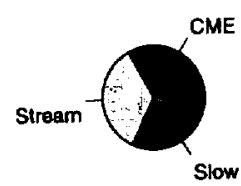

1988-92
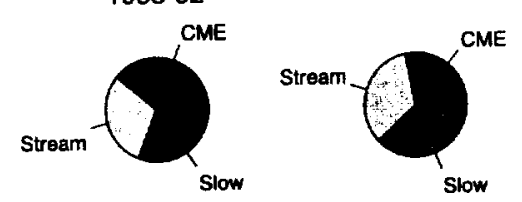

Percent. aa
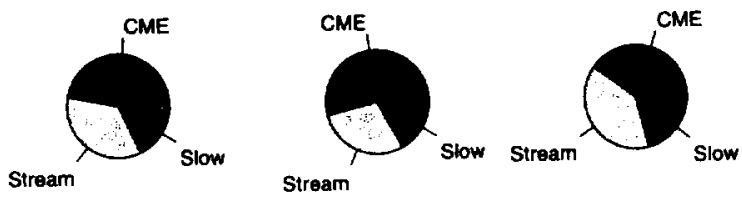

Percent. $|\mathbf{B}|$
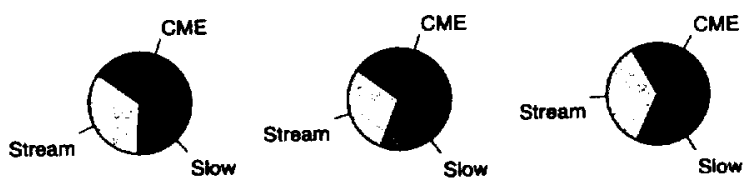

Figure 7. Pie plots showing the fraction of the solar wind occupied by each type of solar wind structure, and the relative contributions to $a a$ and $|B|$, averaged over intervals around (a) solar minimum and (b) solar maximum in 19722000 . Note that the "CME" contribution includes postshock flows in addition to interplanetary coronal mass ejections, also that "unclear" intervals have been removed from the analysis.

to $\overline{a a}$ throughout the solar cycle, but notably contributed more significantly $(\sim 30-40 \%)$ in $1995-1997$ (Figure 2c) during the solar minimum preceding cycle 23 .

[21] The major contribution to the IMF is from highspeed streams at solar minimum ( $\sim 55 \%)$, but from CME- related structures $(\sim 40 \%)$ at solar maximum. We should emphasize however that the "CME-related" contribution to the IMF does not correspond to the magnetic fields added to the solar wind by coronal mass ejections but also includes those fields present during the intervals following CMEdriven shocks when geomagnetic activity is dominated by the effects of the transient flows. (Smith and Phillips [1997] suggested that CMEs added only $\sim 8 \%$ of the magnetic flux at 1 AU near solar maximum, though this may be an underestimate because they identified ICMEs using a limited set of signatures (principally bidirectional solar wind electron heat fluxes) and hence may have missed some events or underestimated the duration of others [e.g., Richardson and Cane, 1995].)

[22] Table 1 shows that averages of the $a a$ index in each type of structure are also relatively constant from cycle to cycle and from minimum to maximum (CME-related 39 nT; high-speed streams $28 \mathrm{nT}$; slow solar wind $14 \mathrm{nT}$ ). Values of $|B|$ are $\sim 9 \mathrm{nT}$ (CME-related), $\sim 7 \mathrm{nT}$ (high-speed streams) and $\sim 6 \mathrm{nT}$ (slow solar wind), with a $\sim 1 \mathrm{nT}$ increase from solar minimum to solar maximum. (Note though that the solar minimum to maximum variation in $|B|$ evident in the 3-rotation averages in Figure 5c is larger than suggested by these values because the solar maximum average is influenced by the Gnevyshev Gap effect.) The average solar wind speeds for the three components $(\sim 460$ $\mathrm{km} / \mathrm{s}$, CME-related; $\sim 500 \mathrm{~km} / \mathrm{s}$, high-speed streams; and $\sim 360 \mathrm{~km} / \mathrm{s}$, slow solar wind) are relatively constant across cycles 21 and 22 and also from solar minimum to solar maximum, the most significant change being a $\sim 50 \mathrm{~km} / \mathrm{s}$ decrease for high-speed streams from solar minimum to solar maximum (Table 1). Thus, even though CMEs are occasionally observed at the Sun moving outwards at speeds much greater than average solar wind speeds, and such events are more frequent near solar maximum, they do not have a major impact on the average speeds of CMErelated structures.

\section{Discussion}

[23] We have examined, for the period 1972-2000, the solar wind structures contributing to extended (e.g., $\sim$ yearly) averages of the $a a$ index $(\overline{a a})$ and solar wind parameters which are typically used in studies of long-term trends in these parameters. We note that such averages are complex since they include contributions from different types of solar wind structures, and the relative contributions of these structures vary during the solar cycle. We find that $\overline{a a}$ and the IMF most closely follow the corresponding averages for corotating high-speed streams through the solar cycle. However, this does not mean that streams dominate $\overline{a a}$ or the IMF throughout the solar cycle. Though streams provide the major contribution $(\sim$ two-thirds) to $\overline{a a}$ at periods of low solar activity levels, and continue to contribute $\sim 30 \%$ around solar maximum, CME-related structures make a significant $(\sim 50 \%)$ contribution around solar maximum. This enhanced CMErelated activity at solar maximum is offset to some extent by the lower activity associated with the slow solar wind. The net result is that $\bar{a} \bar{a}$ tends to intermediate values typically associated with corotating streams. Similar arguments apply for the IMF. 
Table 1. Summary of Time, $a a$, and $\mid B_{1}$ Contributions, and Averages of $a a,|B|$, and the Solar Wind Speed, for Solar Minimum and Maximum Intervals in 1972-2000 (CME-Related Flows: High-Speed Streams: Slow Solar Wind)

\begin{tabular}{|c|c|c|c|c|c|c|}
\hline Interval & Percent Time & Percent $a a$ & Percent B & $a a, \mathrm{nT}$ & $B, \mathrm{nT}$ & $V_{s m}, \mathrm{~km} / \mathrm{s}$ \\
\hline $\begin{array}{l}1973-1977 \\
1983-1987 \\
1993-1997 \\
\text { All }\end{array}$ & $\begin{array}{l}9: 58: 33 \\
9: 53: 37 \\
6: 52: 43 \\
9: 55: 36\end{array}$ & $\begin{array}{c}13: 70: 17 \\
16: 62: 22 \\
9: 68: 23 \\
12: 67: 21\end{array}$ & $\begin{array}{c}\text { Solar Minim } \\
11: 61: 28 \\
10: 53: 37 \\
9: 51: 40 \\
10: 55: 35\end{array}$ & $\begin{array}{l}37,30,13 \\
42,30,15 \\
35,30,12 \\
38,30,13\end{array}$ & $\begin{array}{l}8.0,6.3,5.2 \\
8.6,6.9,5.8 \\
9.2,5.9,4.9 \\
8.5,6.3,5.2\end{array}$ & $\begin{array}{l}445,533,370 \\
460,526,376 \\
413,499,354 \\
438,520,364\end{array}$ \\
\hline $\begin{array}{l}1978-1982 \\
1988-1992 \\
1998-2000 \\
\text { All } \\
\text { Cycle 21 } \\
\text { Cycle 22 } \\
\end{array}$ & $\begin{array}{l}33: 35: 32 \\
39: 30: 31 \\
28: 34: 38 \\
36: 33: 31 \\
23: 44: 33 \\
22: 44: 34 \\
\end{array}$ & $\begin{array}{l}47: 35: 18 \\
54: 29: 17 \\
40: 39: 22 \\
50: 32: 17 \\
33: 48: 19 \\
32: 49: 19\end{array}$ & $\begin{array}{c}\text { Solar Maxim } \\
40: 34: 26 \\
40: 29: 32 \\
33: 35: 32 \\
40: 32: 28 \\
31: 40: 29 \\
22: 42: 36 \\
\end{array}$ & $\begin{array}{l}35,25,14 \\
43,29,16 \\
33,26,13 \\
39,27,15 \\
36,27,14 \\
42,30,14 \\
\end{array}$ & $\begin{array}{l}9.0,7.3,6.4 \\
9.6,7.6,6.9 \\
8.5,6.8,6.0 \\
9.2,7.4,6.6 \\
8.8,7.0,6.1 \\
9.6,6.5,5.7 \\
\end{array}$ & $\begin{array}{l}445,462,355 \\
476,504,366 \\
459,475,366 \\
455,473,358 \\
445,479,360 \\
470,512,363\end{array}$ \\
\hline
\end{tabular}

[24] It is of interest to consider the effects of individual great storms on long-term averages. Figure 1 shows that the average $a a$ values associated with CMEs around solar maximum are typically only relatively modestly enhanced from those in corotating high-speed streams $(\sim 25-50 \mathrm{nT}$ compared with $\sim 20-35 \mathrm{nT}$; see also Table 1), despite the fact that some CMEs generate large storms with $a a \sim$ several hundred $\mathrm{nT}$. Consider for example, the period of Carrington rotations 1965-68 (May 16-August 6, 2000) which has a large contribution to $\overline{a a}$ from CME-related structures $(67 \%)$. This period included the "Bastille Day" event on July 14 which produced a storm with $a a=440 \mathrm{nT}$ when the related postshock flows and ICME (a magnetic cloud) passed Earth, and several other CME-related storms with $a a>100 \mathrm{nT}$. However, aa exceeded $100 \mathrm{nT}$ for only 54 hours $(\sim 3 \%)$ of this 3-rotation interval. The contributions of high-speed streams, slow and "unclear" solar wind to ä were $20 \%, 12 \%$ and $1 \%$, respectively. The mean aa for CME-related structures at this time was $36 \mathrm{nT}$, compared with 22,17 and $24 \mathrm{nT}$, respectively for the other structure types, while the percentage of the time associated with each type of structure was $52 \%, 26 \%, 21 \%$, and $2 \%$, respectively. Combining the mean $a a$ values and times gives an average $a a$ for all solar wind structures of $\sim 28 \mathrm{nT}$. Note that this average is closer to the activity level associated with corotating streams $(22 \mathrm{nT})$ than to the $36 \mathrm{nT}$ associated with CMEs, even though CMEs make a larger contribution to the average $a a$ than the other structure types and several major storms were present. Thus, the major storms have relatively little impact on the 3-rotation average of $a a$ because they are short-lived.

[25] To investigate the variation in geoeffectiveness of streams and other structures over the solar cycle, we have also examined the variations in the solar wind speed, IMF strength and $V^{2} B_{s}$. Considering the IMF strength (which is essentially proportional to $B_{s}$ ), we find that the enhancement as solar activity levels increase is seen clearly in the largescale, long-lived structures (corotating streams and slow interstream solar wind) and to a lesser degree in CMErelated structures. This indicates that the enhancement is not solely caused by the magnetic flux added by the increasing number of transients as activity levels increase. Thus, longterm averages of the IMF provide information on the background field in the heliosphere through which the transients are propagating. Consistent with this, we note a close association between variations in $|B|, a a$ and the open flux at the Sun, in particular around solar maximum. Both the solar wind speed and IMF strength show evidence of temporary decreases for periods of $<\sim 1$ year right at solar maximum, i.e. the "Gnevyshev Gap". Overall, however, there is a rather poor correlation between $a a$ and the IMF strength throughout the solar cycle, mainly because of the high activity levels associated with high-speed streams when fields are weak around solar minimum. The variation of $V^{2} B_{s}$ shows a much higher correlation with $a a$ than either of its components, both in all solar wind and in individual classes of structures.

[26] Acknowledgments. H.V.C. was supported at GSFC by a contract with USRA. I.G.R. was supported by NASA grant NCC 5-180. We wish to thank Y.-M. Wang (Naval Research Laboratory) for the open flux data, and A. Ling for computational support. We acknowledge the use of OMNI nearEarth solar wind data supplied by the National Space Science Data Center and ACE data supplied by the ACE Science Center. The a a data were obtained from the NOAA National Geophysical Data Center and from the Service Intemational des Indices Geomagnetiques.

[27] Janet G. Luhmann thanks Paul Gazis and another referee for their assistance in evaluating this paper.

\section{References}

Ahluwalia, H. S., Three cycle quasiperiodicity in solar wind from polar coronal holes and the size of solar activity cycle 23, in Solar Wind Nine, edited by S. R. Habbal et al., AIP Conf. Proc., 471, $415,1999$.

Belcher, J. N., and L. Davis Jr., Large-amplitude Alfvén waves in the interplanetary medium, 2, J. Geophys. Res., 76, 3534, 1971.

Bounar, K. H., E. W. Cliver, and V. Boriakoff, A prediction of the peak sunspot number for solar cycle 23, Sol. Phys., 176, $211,1997$.

Cane, H. V., I. G. Richardson, T. T. von Rosenvinge, and G. Wibberenz, Cosmic ray decreases and shock structure: A multispacecraft study, $J$. Geophys. Res., 99, 21,429, 1994.

Cane, H. V., G. Wibberenz, I. G. Rjchardson, and T. T. von Rosenvinge, Cosmic ray modulation and the solar magnetic field, Geophys. Res. Lett. $26,565,1999$.

Cane, H. V., I. G. Richardson, and O. C. St. Cyr, Coronal mass ejections. interplanetary ejecta and geomagnetic storms, Geophys. Res. Lett., 27, 3591, 2000.

Cliver, E. W., V. Boriakoff, and K. H. Bounar, Gcomagnetic activity and the solar wind during the Maunder Minimum, Geophys. Res. Lett., 25, 897. 1998.

Crooker, N. U., and E. W. Cliver, Postmodem view of M-regions, J. Geophys. Res., 99, 23,383, 1994.

Feminella, F., and $M$. Storini, Large scale dynamical phenomena during solar activity cycles, Astron. Astrophys., 322, 311, 1997

Feynman, J., Implications of solar cycles 19 and 20 geomagnetic activity for magnetospheric processes, Geophys. Res. Lett., 7, 971, 1980.

Feynman, J., and N. U. Crooker, The solar wind at the turn of the century, Nature, 275, 626, 1978. 
Gnevyshev, M. N., On the 11-years cycle of solar activity, Sol. Phys, l, $107,1967$.

Gnevyshev, M. N., Essential features of the 11 year solar cycle, Sol. Phys., 5l, $175,1977$.

Gosling, J. T., Coronal mass ejections and magnetic flux ropes in interplanetary space, in Physics of Magnetic Flux Ropes, Geophys. Monogr. Ser. vol. 58, edited by C. T. Russcll, E. R. Priest, and L. C. Lee, p. 343, AGU, Washington, D. C., 1990.

Gosling, J. T., D. N. Baker, S. J. Bame, W. C. Feldman, R. D. Zwickl, and E. J. Smith, Bidircctional solar wind clectron heat flux events, J. Geophys. Res. 92, 8519, 1987

Gosling. J. T., D. J. McComas, J. L. Phillips, and S. J. Bame, Geomagnetic activity associated with Earth passage of interplanetary shock disturbances and coronal mass cjections, J. Geophys. Res., 96, 7831, 1991.

Hathaway, D. H., R. M. Wilson, and E. J. Reichmann, A synthesis of solar cycle prediction techniques, J. Geophys. Res., 104, 22,375, 1999.

Hundhausen. A. J., An interplanetary view of coronal holes, in Coronal Holes and High Speed Wind Streams, cdited by J. B. Zirker, p. 225, Colo. Assoc. Univ. Press, Boulder, 1977.

Kishcha, P. V., I. V. Dinitrieva, and V. N. Obridko, Long-term variations of the solar-geomagnetic correlation, total solar radiance, and Northern Hemispheric tempcrature, J. Atmos. Sol. Terr. Phys., 61, 799, 1999.

Lindsay, G. M., J. G. Luhmann, C. T. Russell, and J. T. Gosling, Relationship between coronal mass ejection speeds from coronagraph images and interplanetary characteristics of the associated interplanetary coronal mass cjections, $J$. Geophys. Res., 104, 12,515, 1999.

Lockwood, M., Long-term variations in the magnetic field of the Sun and the heliosphere: The origin, effects, and implications, J. Geophys. Res., 106, 16,021, 2001 .

Lockwood, M., and S. Foster, Long-term yariations in the magnetic field of the Sun and possible implications for terrestrial climate, in The Solar Cycle and Terrestrial Climate, edited by A. Wilson, Eur. Space Agency Spec. Publ., ESA SP-463, 85, 2000.

Lockwood, M., R. Stamper, and M. N. Wild, A doubling of the solar magnetic field during the last 100 years, Nature, 399, 437, 1999.

Luhmann, J. G., Y. Li, C. N. Arge, P. R. Gazis, and R. Ulich, Solar cycle changes in coronal holes and space weather cycles, J. Geophys. Res., 107, 10.1029/2001 JA007550, in press, 2002.
Mayaud, P. N., The $a a$ indices: A 100-year series characterising the geomagnctic activity, J. Geophys. Res., 77, 6870, 1972.

Richardson, I. G., and H. V. Cane, Regions of abnormally low proton remperature in the solar wind (1965-1991) and their association with ejecta, J. Geophys. Res., 100, 23,397, 1995.

Richardson, I G. J. E. Mazur, and G. M. Mason, A comparison of recurrent energetic ion cnhancements observed at Ulysses and at 1 AU by IMP 8 and SAMPEX: Ulysses launch until following the first north polar passage, J. Geophys. Res., 103, 2115, 1998.

Richardson, I. G., E. W. Cliver, and H. V. Cane, Sources of geomagnetic activity over the solar cycle: Relative importance of CMEs, high-speed streams, and slow solar wind, $J$. Geophys. Res., 105, 18,203, 2000.

Richardson, I G. F W. Cliver, and H. V. Cane, Sources of geomagnetic storms for solar minimum and maximum conditions during 1972-2000, Geophys. Res. Lett., 28, 2569, 2001.

Slavin, J. A., and E. J. Smith, Solar cycle variations in the interplanetary magnetic field: Solar Wind 5 , edited by M. Neugebaucr, NASA Conf Publ., 2280, 323, 1983.

Smith, C. W. and J. L. Phillips, The role of CMEs and interplanetary shocks in IMF statistics and solar magnetic flux ejection, $J$. Geophys. Res., 102, 249, 1997.

Stamper, R., M. Lockwood, M. N. Wild, and T. D. G. Clark, Solar causes of the long-term increase in geomagnetic activity, J. Geophys. Res., 104, $28,325,1999$.

Tsurutani, B. T., W. D. Gonzalcz, A. L. C. Gonzalez, F. Tang, J. K. Arballo, and M. Okada. Interplanetary origin of geomagnetic activity in the declining phase of the solar cycle, J. Geophys Res., 100, 21,717, 1995.

Wang, Y.-M., S. H. Hawley, and N. R. Sheeley Jr., The magnetic nature of coronal holcs, Science, 271, 464, 1996.

Wang, Y. M., J. Lean, and N. R. Sheelcy Jr., The long-term variation of the Sun's open magnctic flux, Geophys. Res. Lett., 27, 505, 2000.

H. V. Cane and 1. G. Richardson, Code 661, Laboratory for High Energy Astrophysics, NASA Goddard Space Flight Center, Greenbelt, MD 20771 , USA. (hilary.cane@utas.edu.au; richardson@lheavx.gsfc.nasa.gov)

E. W. Cliver, Air Force Research Laboratory, Hanscom AFB, MA 01731, USA. (cliver@plh.af.mil) 
